\title{
XYLAMINE ENHANCES PINEAL GLAND $N$-ACETYLTRANSFERASE ACTIVITY IN VITRO
}

\author{
Katherine A. Haak, ${ }^{1}$ German Torres, ${ }^{1}$ Patricia L. Garvey, ${ }^{2}$ \\ David M. Bronstein, ${ }^{3}$ Arthur K. Cho ${ }^{4}$ and Loy D. LyTle ${ }^{1}$ \\ 'Laboratory of Psychopharmacology, Department of Psychology, University of California, \\ Santa Barbara, CA 93106, \\ ${ }^{2}$ International Ophthalmic, Laguna Hills, CA 92651, \\ ${ }^{3}$ Department of Psychiatry, Mental Health Research Institute, University of Michigan, Ann Arbor, \\ MI 48109 and \\ ${ }^{4}$ Department of Pharmacology, UCLA School of Medicine, Center for the Health Sciences, Los Angeles, \\ CA 90024, U.S.A.
}

(Received 26 February 1988)

\begin{abstract}
The action of $N$-2'-chloroethyl- $N$-ethyl-2-methyl benzylamine (xylamine) on rat pineal gland sympathetic innervation was examined.

2. This alkylating agent caused a concentration-dependent increase in pineal gland $N$-acetyltransferase (NAT) activity in neurologically intact pineal glands that was suppressed in glands previously subjected to bilateral superior cervical ganglionectomy.

3. Xylamine-induced elevations in NAT activity were attenuated by $\beta$-noradrenergic antagonist drugs but not by $\alpha$-noradrenergic antagonist drugs.

4. Since pineal gland uptake of radiolabelled norepinephrine (NE) was impaired by xylamine, the drug may increase pineal gland NAT activity by inhibiting NE reuptake into the presynaptic nerve terminal, thereby increasing the amount of the neurotransmitter available to stimulate pinealocyte $\beta$-noradrenoceptors.
\end{abstract}

\section{INTRODUCTION}

Xylamine $\left(N-2^{\prime}\right.$-chloroethyl- $N$-ethyl-2-methyl benzylamine) appears to be an irreversible inhibitor of neuronal norepinephrine (NE) uptake in both central (Dudley et al., 1981) and peripheral nerves (Cho et al., 1980; Fischer and Cho, 1982; Ransom et al., 1984, 1985). The aziridinium ion, resulting from the intramolecular cyclization of xylamine, is believed to mediate this inhibitory effect on neuronal uptake by alkylating the NE uptake carrier molecule (Ransom et al., 1982, 1984). A great deal has been learned recently about the neurochemical bases of xylamine-induced alterations in noradrenergic neurotransmitter mechanisms, but relatively little is known about the possible functional consequences of these actions.

The present experiments were undertaken to study the possible functional effects of xylamine on a set of well-characterized noradrenergic synapses known to influence the synthesis of the pineal gland hormone melatonin. The pineal gland enzyme, serotonin $N$-acetyltransferase (NAT; EC 2.3.1.5) plays an important role in the formation of melatonin.

Norepinephrine molecules released from postganglionic, sympathetic neurons innervating the pineal gland enhance the activity of NAT via stimulation of a $\beta$-noradrenoceptor linked, cyclic AMP dependent mechanism (Axelrod, 1974). Pharmacological manipulations, including those involving such well known noradrenergic neurotransmitter uptake inhibitors as desmethylimip- ramine and amphetamine, that increase synaptic $\mathrm{NE}$ levels, elevate pineal gland NAT activity (Axelrod, 1974; Klein, 1979; Reiter, 1981). In the present experiments we determined whether xylamine might also increase pineal gland NAT activity via noradrenergic mechanisms similar to those known to be important for other indirectly acting noradrenergic neurotransmitter agonists.

\section{MATERIALS AND METHODS}

\section{Animals}

Male Sprague-Dawley albino rats, weighing $250-350 \mathrm{~g}$, were either purchased from Charles River Breeding Laboratories (Wilmington, MA) or bred and reared in our animal colony. Animals were housed in groups of 5-6 in hanging wire cages, given ad libitum access to tap water and laboratory chow (Ralston Purina, St Louis, MO), maintained under $22-23^{\circ} \mathrm{C}$ ambient temperature conditions at $55 \%$ relative humidity, and exposed to a $12: 12 \mathrm{hr}$ light:dark cycle (lights on at $0700 \mathrm{hr}$; incandescent illumination was approx. $32 \mathrm{~lx}$ during the light phase).

All experiments were conducted during the middle of the light phase of the cycle. Animals were killed by decapitation and pineal glands were excised within 1-2 min. Pineal glands were immediately placed in BGJb Fritton-Jackson culture medium (Grand Island Biological Co., Grand Island, NY) and blood vessels and connective tissue were removed with the aid of a dissection microscope prior to in vitro culture.

\section{Chemicals}

Xylamine $\mathrm{HCl}$ was synthesized as described by Kammerer et al. (1979). A $100 \mu \mathrm{M}$ solution of xylamine $\mathrm{HCl}$ was 
prepared in $10 \mathrm{mM}$ sodium phosphate buffer (pH 7.4) and allowed to stand at least $30 \mathrm{~min}$ at room temperature prior to use. Yohimbine $\mathrm{HCl}$ was purchased from Sigma Chemical Co. (St Louis, MO), and all other drugs used in these experiments were generously provided by the following companies: prazosin $\mathrm{HCl}$ (Pfizer Inc., Groton, CT); desmethylimipramine $\mathrm{HCl}$ (DMI; Revlon Health Care Group, Tuckahoe, NY), butoxamine $\mathrm{HCl}$ (Burroughs Wellcome Co., Research Triangle Park, NC) and practolol $\mathrm{HCl}$ (Imperial Chemical Industries Ltd, Alderley Park, Cheshire, England).

\section{Effect of xylamine on pineal gland and NAT activity}

For in vitro experiments, pineal glands were placed in organ culture using a modification (Altar et al., 1983) of the method of Parfitt et al. (1976). In brief, pineal glands were preincubated for $30 \mathrm{~min}$ in $247 \mu$ l of incubation medium at $37^{\circ} \mathrm{C}$ in a $95: 5 \mathrm{O}_{2}: \mathrm{CO}_{2}$ atmosphere. $13 \mu \mathrm{l}$ of either vehicle or one of several different concentrations of the drug were added to the incubation medium and $4 \mathrm{hr}$ later glands were frozen and stored at $-70^{\circ} \mathrm{C}$

Pineal gland NAT activity was measured using the protocol described by Altar et al. (1983) based on the method of Deguchi and Axelrod (1972). Changes in pineal gland NAT activity, calculated as the nmoles of $N$-acetyltryptamine formed per pineal per $\mathrm{hr}$, were analyzed using analysis of variance followed by post hoc Student's $t$-test.

\section{Effect of xylamine on NAT activity in denervated pineal glands}

In order to determine whether the possible xylamineinduced alterations in NAT activity were mediated by preor post-synaptic mechanisms, pineal glands obtained from denervated or neurologically intact animals were incubated with vehicle or xylamine. Denervations were accomplished by bilateral superior cervical ganglionectomies (SCGX) performed under ether anesthesia 7 days prior to placing the glands in organ culture. Successful removal of the ganglia was assessed in vivo by the appearance of ptosis.

\section{Xylamine inhibition of $\left[{ }^{3} \mathrm{H}\right] \mathrm{NE}$ uptake}

Previous studies indicated that xylamine effectively blocks neuronal reuptake of NE (Cho et al., 1980; Ransom et al., 1985). We determined whether xylamine might affect norepinephrine uptake by using a modification of the method of Bowers et al. (1984). Briefly, pineal glands were placed in organ culture and coincubated with vehicle, $100 \mu \mathrm{M}$ xylamine, or the well-known NE reuptake inhibitor DMI $(100 \mu \mathrm{M})$. After a $1 \mathrm{hr}$ preincubation period glands were transferred to wells containing $1.2 \times 10^{-7} \mathrm{M}$ $\left[{ }^{3} \mathrm{H}\right]-1$-norepinephrine (specific activity $=19.7 \mathrm{Ci} / \mathrm{mmol}$, New England Nuclear Corp., Boston, MA) as well as the appropriate drug or vehicle solution. At the end of the $15 \mathrm{~min}$ incubation period the glands were washed in Earle's salt solution, and the amount of $\left[{ }^{3} \mathrm{H}\right] \mathrm{NE}$ in the glands was determined by liquid scintillation spectrometry.

\section{Blockade of xylamine-induced increase in NAT activity}

In addition to the importance of $\beta$-noradrenoceptor stimulation for pineal gland NAT activity, $\alpha$-noradrenoceptors may also play a permissive role in the noradrenergic induction of the enzyme (Klein et al., 1983; Alphs and Lovenberg, 1984). In order to more precisely characterize the receptor subtype which might mediate xylamine-induced changes in NAT activity, pineal glands were coincubated with xylamine and $100 \mu M$ practolol $\mathrm{HCl}$ or butoxamine $\mathrm{HCl}\left(\beta_{1}-\right.$ and $\beta_{2}$-noradrenoceptor antagonist drugs, respectively) or prazosin $\mathrm{HCl}$ or yohimbine $\mathrm{HCl}\left(\alpha_{1}\right.$ - and $\alpha_{2}$-noradrenoceptor antagonist drugs, respectively).

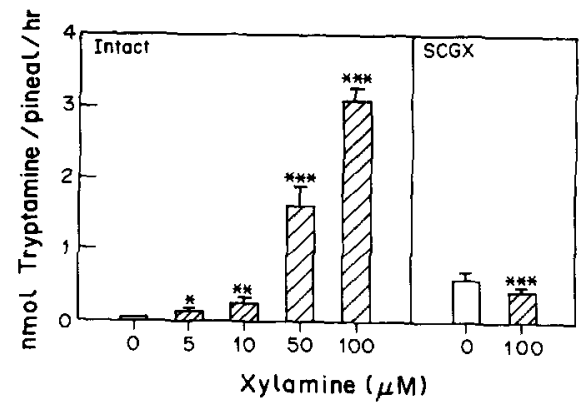

Fig. 1. Effect of xylamine on in vitro NAT activity in intact or SCGX denervated pineal glands placed in organ culture. All values are means $( \pm$ SEM $) \quad(N=4-5$ animals per group). The left panel represents the number of nmole acetyltryptamine/pineal gland/hour in intact rats $\left(^{*} P<0.02 ;{ }^{* * P}<0.01 ;{ }^{* * *} P<0.001\right.$ compared to intact vehicle treated control animals). The right panel represents acetyltryptamine formation in pineal glands from bilaterally superior cervical ganglionectomized (SCGX) rats $\left({ }^{* * *} P<0.001\right.$ as compared to intact xylamine treated animals).

\section{RESULTS}

Xylamine caused a concentration dependent increase in pineal gland NAT activity (Fig. 1). At the lowest concentration tested $(5 \mu \mathrm{M})$ xylamine produced a 2 -fold increase in NAT activity whereas at its highest concentration $(100 \mu \mathrm{M})$ the activity of the enzyme was elevated 77-fold. Although xylamine increased NAT activity in neurologically intact pineal glands, its in vitro action was substantially attenuated in pineal glands obtained from animals previously subjected to SCGX (Fig. 1).

Neurologically intact pineal glands took up approximately $0.30 \mathrm{pmol}$ of $\left[{ }^{3} \mathrm{H}\right] \mathrm{NE}$ from the incubation media during the $15 \mathrm{~min}$ test period. Interestingly, both xylamine and DMI caused an $80-90 \%$ inhibition of $\left[{ }^{3} \mathrm{H}\right] \mathrm{NE}$ uptake into these glands (Fig. 2).

When administered alone none of the $\alpha$ - or $\beta$-noradrenoceptor antagonist drugs produced any

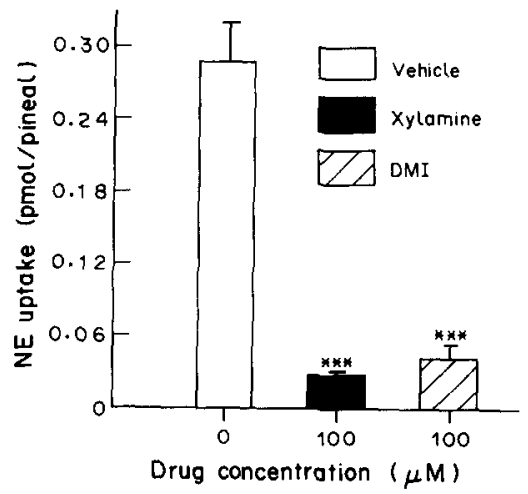

Fig. 2. Effects of xylamine or DMI on in vitro pineal gland $\left[{ }^{3} \mathrm{H}\right] \mathrm{NE}$ uptake. Pineal glands were incubated with $\left.{ }^{3} \mathrm{H}\right] \mathrm{NE}$ for $15 \mathrm{~min}$ in the presence of vehicle, xylamine or DMI. All values are mean NE uptake $( \pm$ SEM $)(N=5$ animals per group; ${ }^{* * *} P<0.001$ compared to vehicle treated control animals). 
Table 1. Effects of $\alpha$ - or $\beta$-noradrenoceptor antagonist drugs on xylamine induced increases in pineal gland NAT activity

\begin{tabular}{lcc}
\hline & $\begin{array}{c}\text { NAT activity } \\
\text { Vehicle }\end{array}$ & $\begin{array}{c}\text { Xylamine } \\
\text { Drug conditon }\end{array}$ \\
\hline Vehicle & $0.02 \pm 0.01$ & $2.24 \pm 0.05^{*}$ \\
Practolol & $0.02 \pm 0.01$ & $0.07 \pm 0.02 \dagger^{+}$ \\
Butoxamine & $0.02 \pm 0.01$ & $0.29 \pm 0.06^{*}+$ \\
Prazosin & $0.01 \pm 0.01$ & $2.34 \pm 0.60^{*}$ \\
Yohimbine & $0.01 \pm 0.01$ & $2.19 \pm 0.63^{*}$ \\
\hline
\end{tabular}

Pineal glands ( $N=4$ per group) were coincubated with either vehicle or xylamine $(100 \mu \mathrm{M})$ and $\alpha$-or $\beta$-noradrenoceptor antagonist drugs $(100 \mu \mathrm{M})$. All values are the means ( \pm SEM) nmoles of $N$-acetyltryptamine formed/pineal gland $/ \mathrm{hr}$ $(* P<0.01$ compared to vehicle: vehicle treated control values; $+P<0.01$ compared to vehicle:xylamine treated control).

significant changes in pineal gland NAT activity compared to vehicle control values (Table 1). However, each of the $\beta$-noradrenoceptor but none of the $\alpha$-noradrenoceptor antagonist drugs significantly attenuated the xylamine-induced elevations in pineal gland NAT activity.

\section{DISCUSSION}

Xylamine in vitro enhanced pineal gland NAT activity in a concentration dependent manner. This action of the drug is abolished in denervated glands and is attenuated by $\beta$ - but not $\alpha$-noradrenoceptor antagonist drugs. Since the abilities of neurologically intact pineal glands to accumulate $\left[{ }^{3} \mathrm{H}\right] \mathrm{NE}$ are substantially reduced by xylamine, our combined data suggest that xylamine elevates pineal gland NAT activity by increasing synaptic NE concentrations at pinealocyte $\beta$-receptors via the inhibition of neurotransmitter uptake into postganglionic presynaptic noradrenergic nerve terminals. Previous studies suggest that rat vas deferens presynaptic noradrenergic nerve terminals accumulate xylamine (Ransom et al., 1985). Chemical denervation of the terminals by 6-hydroxydopamine depletes NE content by $85 \%$ and reduces radiolabelled xylamine accumulation by $70 \%$. Since the NAT response to xylamine is also abolished in denervated pineal glands, it appears that xylamine increases pineal gland NAT activity by acting presynaptically on one or more noradrenergic neurotransmitter mechanisms.

The NE uptake inhibiting properties of xylamine have been described previously in rabbit aortic rings (Cho et al., 1980) as well as in rat superior cervical ganglia (Fischer and Cho, 1982), vas deferens (Ransom et al., 1985) and cortical synaptosomes (Dudley et al., 1981). Our data indicate that xylamine also inhibits uptake of the neurotransmitter in postganglionic sympathetic terminals innervating the pineal gland. Nevertheless, these results do not rule out other possible mechanisms of action of xylamine. For example, xylamine and its chemical analogue $N$-(2-chloroethyl)- $N$-ethyl-2bromobenzylamine (DSP4) appear to exert neurotoxic effects on peripheral and central noradrenergic nerve terminals (Dudley et al., 1981; Jaim-Etcheverry and Zieher, 1983), Xylamineinduced neurotoxic effects on peripheral catecholaminergic nerves still remain to be determined, however, since only marginal NE depletions in the rat vas deferens have been observed previously (Ransom et al., 1984, 1985). Nevertheless, xylamine might also increase synaptic NE by other nontoxic mechanisms, such as by increasing release of the neurotransmitter from sympathetic nerve terminals via an amphetamine-like action on the noradrenergic nerve terminals that adhere to the pineal gland when it is placed in organ culture.

Thus far xylamine has proven to be useful in examinations of the molecular mechanisms important for neuronal NE uptake. Although its neurochemical mechanisms of action appear to differ significantly from some of the other noradrenergic reuptake inhibiting drugs available in the experimental and clinical armamentarium, it remains to be determined whether these mechanisms will prove useful as a tool for gaining a better understanding of the functional importance of noradrenergic neurons in various in vivo or in vitro preparations.

Acknowledgement - This research was supported in part by NIMH grant MH-31134 to L.D.L.

\section{REFERENCES}

Alphs L. and Lovenberg W. (1984) Modulation of rat pineal acetyl-CoA: arylamine $N$-acetyltransferase induction by alpha adrenergic drugs. J. Pharmac. exp. Ther. 230, 431-437.

Altar A., Motroni T. P. and Lytle L. D. (1983) Functional synaptogenesis and the rat pineal gland: a pharmacological investigation. J. Neural Transm. 58, 231-241.

Axelrod J. (1974) The pineal gland: a neurochemical transducer. Science N.Y. 184, 1341-1348.

Bowers C. W., Baldwin C. and Zigmond R. E. (1984) Sympathetic reinnervation of the pineal gland after postganglionic nerve lesion does not restore normal pineal function. J. Neurosci. 4, 2010-2015.

Cho A. K., Ransom R. W., Fischer J. B. and Kammerer R. C. (1980) The effects of xylamine, a nitrogen mustard, on $\left[{ }^{3} \mathrm{H}\right]$ norepinephrine accumulation in rabbit aorta. J. Pharmac. exp. Ther. 214, 324-327.

Deguchi T. and Axelrod J. (1972) Sensitive assay for serotonin $N$-acetyltransferase activity in rat pineal. Analyt. Biochem. 50, 174-179.

Dudley M. W., Butcher L. L., Kammerer R. C. and Cho A. K. (1981) The actions of xylamine on central noradrenergic neurons. J. Pharmac. exp. Ther. 217, 834-840.

Fischer J. B. and Cho A. K. (1982) Inhibition of $\left[{ }^{3} \mathrm{H}\right]$ norepinephrine uptake in organ cultured rat superior cervical ganglia by xylamine. J. Pharmac, exp. Ther. 220, 115-119.

Jaim-Etcheverry G. and Zieher L. M. (1983) 2-chloroethylamines: new chemical tools for the study of noradrenergic neurons. Trends Pharmac. Sci. 4, 473-475.

Kammerer R. C., Amiri B. and Cho A. K. (1979) Inhibition of uptake of catecholamines by benzylamine derivatives. J. med. Chem. 22, 352-355.

Klein D. C. (1979) Circadian rhythms in the pineal gland. In Endocrine Rhythms (Edited by Krieger D. T.), pp. 203-220. Raven Press, New York.

Klein D. C., Sugden D. and Weller J. (1983) Postsynaptic alpha-adrenergic receptors potentiate the beta-adrenergic stimulation of pineal serotonin $N$-acetyltransferase. Proc. natn. Acad. Sci U.S.A. 80, 599-603.

Parfitt A., Weller J. L. and Klein D. C. (1976) Betaadrenergic blockers decrease adrenergically stimulated $\mathrm{N}$-acetyltransferase activity in pineal glands in organ chamber. Neuroendocrinology 15, 353-358. 
Ransom R. W., Kammerer R. C. and Cho A. K. (1982) Chemical transformations of xylamine ( $N-2^{\prime}$-chloroethyl$N$-ethyl-2-methylbenzylamine) in solution. Molec. Pharmac. 21, 380-386.

Ransom R. W., Waggaman L. A. and Cho A. K. (1984) $\left[{ }^{3} \mathrm{H}\right]$ Xylamine accumulation by two sympathetically innervated tissues. $J$. Neurochem. 42, 475-481.
Ransom R. W., Waggaman L. A. and Cho A. K. (1985) Interaction of xylamine with peripheral sympathetic neurons. Life Sci. 37, 1177-1182.

Reiter R. J. (1981) The mammalian pineal gland: structure and function. Am. J. Anat. 162, 287-313. 